\title{
Forschung \& Entwicklung an Fachhochschulen in Österreich: Leistung und Erfolgsgeschichte trotz herausfordernder Rahmenbedingungen?
}

\author{
G. Sabbatini, J. Kastner
}

\begin{abstract}
In diesem Übersichtsbeitrag wird die Forschungs- und Entwicklungsleistung der österreichischen Fachhochschulen aus Sektor-interner Sicht dargestellt. Neben quantitativen und qualitativen Angaben zur Entwicklung der letzten Jahre werden die dazugehörigen Rahmenbedingungen und deren Auswirkungen auf den Aufbau der Forschungsaktivitäten im Fachhochschulsektor kritisch überprüft. Aktuelle Aussichten sowie mögliche Empfehlungen aus Sicht der Autoren runden das Bild ab.
\end{abstract}

Schlüsselwörter: Fachhochschulen; Forschung

\section{Research \& development at Austrian Universities of Applied Sciences: a success story despite challenging framework conditions?}

In this overview, the achievements of the Austrian Universities of Applied Sciences in research \& development (R\&D) are analyzed from a point of view inside the sector. Besides hard and soft facts about the development of the last years, framework conditions and their effects on the structure of R\&D activities in the UAS sector are critically revised. Current perspectives and some suggestions from the point of view of the authors complete the analysis.

Keywords: Universities of Applied Sciences; research

Eingegangen am 3. September 2019, angenommen am 23. Oktober 2019, online publiziert am 17. Dezember 2019 (c) The Author(s) 2019

\section{Einleitung und Ziel}

In diesem Übersichtsbeitrag möchten wir die Leistung der österreichischen Fachhochschulen (FHs) hinsichtlich Forschung und Entwicklung (F\&E) darstellen und analysieren. Einige Studien, vor allem im Auftrag von Ministerien und Fördergebern, haben sich in den letzten Jahren dieser Frage gewidmet, jedoch halten wir es für wichtig, folgende Fragen aus Sektor-interner Sicht zu erörtern:

- Welche Leistung konnten die österreichischen FHs in Punkto F\&E in ihrer noch relativ kurzen Geschichte aufbauen?

- Wie schauen die Rahmenbedingungen dieser Leistung aus und wie entwickeln sie sich derzeit weiter?

- Wie ist der Zusammenhang zwischen Rahmenbedingungen und Leistung kritisch zu interpretieren, und welche Verbesserungen wären in Zukunft sinnvoll?

\subsection{Ausgangslage und Motivation}

Parallel zum Auftrag zur akademischen Lehre ist der fachhochschulische Auftrag zur angewandten Forschung im Fachhochschulstudiengesetz (FHStG) bereits in seiner ersten Fassung von 1993 verankert. Die Erhalter von FHs sind dazu verpflichtet, die Mitarbeit von Lehre- und Forschungspersonal an F\&E Projekten sicherzustellen. Nichtdestotrotz, während für die Lehre ein Bundesfinanzierungsmodell vom Anfang an umgesetzt wurde, blieb und bleibt nach wie vor eine nachhaltige Bundesfinanzierung der Forschung aus. Bundesmitteln werden den FHs für Forschung lediglich projektbezogen im
Wettbewerb mit anderen Forschungsinstitutionen über die Förderagenturen des Bundes bereitgestellt. Angesichts dieser Ausgangslage zeigt sich im FH-Sektor nach wie vor ein erstaunliches Ausmaß und Wachstum an F\&E Aktivitäten, die wir in diesem Beitrag übersichtlich darstellen möchten.

In den letzten Jahren haben sich bereits mehrere Studien diesem Thema gewidmet (z.B. $[4,5])$, allerdings finden wir wichtig, eine Sektor-interne Sicht hier anzubieten, einerseits um die Daten aus den statistischen Quellen mit Eigenerhebungen des Sektors zu ergänzen, andererseits um die Rahmenbedingungen von F\&E an FHs kritisch zu hinterfragen und Zusammenhänge zwischen Rahmenbedingungen und Entwicklungen zu beleuchten.

\section{2 Überblick}

Der gerade 25 Jahre alte österreichische Fachhochschulsektor besteht im Jahr 2018 aus 21 Institutionen, die im Studienjahr 2017/18 insgesamt über 50.000 Studierende ausbilden, ${ }^{1}$ somit ca. $15 \%$ der Gesamtstudierendenanzahl in Österreich. Die Arbeitslosenrate der AbsolventInnen liegt bei ca. 1,70\%.2

${ }^{1}$ Quelle: Statistik Austria, STATcube

${ }^{2}$ Quelle: Daten der Fachhochschulkonferenz (FHK), eigene Erhebungen.

Sabbatini, Giuliana, Fachhochschule Technikum Wien, Höchstädtplatz 6, 1200 Wien Österreich (E-Mail: giuliana.sabbatini@technikum-wien.at); Kastner, Johann, Fachhochschule Oberösterreich, Roseggerstraße 15, 4600 Wels, Österreich 
Das Hauptunterscheidungsmerkmal zwischen Fachhochschulen und traditionellen Universitäten in Österreich liegt in der Anwendungsorientierung, sowohl in der Lehre als auch in der Forschung. Wir bevorzugen - natürlich nicht nur den FHs vorbehalten! - den Begriff der "Relevanzorientierung". Zum einen, weil die Grenzen zwischen Grundlagen und Anwendung in mehreren Disziplinen und vor allem auf interdisziplinären Gebieten oft verschwimmen. Zum anderen, weil "relevanzorientiert" mehr als Anwendung bedeutet: Die Forschung ist bereits bei der Formulierung ihrer Fragestellungen von deren Relevanz für potenzielle Anwendungsfelder geleitet, aber nicht nur - auch Relevanz für Methoden und Verfahren kann hier ein Kriterium sein, unabhängig vom jeweiligen Anwendungsgebiet. In diesem Zusammenhang lässt sich die zeitliche Komponente in der Perspektive der jeweiligen Forschung als Unterscheidungsmerkmal zwischen Universitäten und FHs identifizieren: kurz- bis mittelfristige Relevanzorientierung an den FHs, mittel- bis langfristig an den Universitäten. ${ }^{3}$ In der Praxis zeigen sich oft fließende Übergänge, so zum Beispiel auch im erfolgreichen Fördermodell des Bundes für Exzellenz an Fachhochschulen, die sogenannten Josef Ressel Zentren (JRZ) der Christian Doppler Gesellschaft (CDG).

In der Identität und Geschichte der Fachhochschulen implizit ist auch die wesentliche Rolle der sogenannten "third mission" als vielfältige (und nicht lineare) Verlinkung zwischen Hochschule, Gesellschaft und Wirtschaft, mit dem Ziel, Forschung, Entwicklung und Innovation als ein Ganzes und die drei Prozesse eng ineinander und mit der Lehre verzahnt zu gestalten.

Bezogen auf F\&E, zeigt sich trotzdem bei näherer Betrachtung ein sehr heterogenes Bild im FH-Sektor. Je nach Bundesland, GröBe, strategischer Ausrichtung und Fachspektrum, spielt die F\&E Positionierung eine unterschiedliche Rolle in der Profilbildung der jeweiligen Institution, sowie im Zusammenspiel zwischen Lehre und Forschung.

Wozu dient, über die gesetzliche Verpflichtung hinaus, F\&E an FHs?

- In erster Linie der Personalentwicklung und Weiterqualifikation im akademischen Bereich. Dies spielt auch bei Attraktivität und Bindung von guten Lehrenden eine große Rolle.

- In Anlehnung an den ersten Punkt, dient F\&E der Aktualität und Weiterentwicklung der Lehre und ihrer Inhalte, sowie der Ausgestaltung von innovativen Ausbildungsangeboten. Auf mehreren Fachgebieten wäre eine Ausbildung auf akademischem Niveau nicht denkbar (bzw. über mehrere Jahre nicht haltbar), wenn das Lehrpersonal nicht laufend in F\&E Projekten tätig wäre.

- Zusammen betrachtet, bedeutet dies mehr Sichtbarkeit nach AuBen, für potenzielle Studierende, Mitarbeiterlnnen und Partner gleichermaßen.

- Darüber hinaus sind die F\&E Aktivitäten im Zusammenspiel mit Wirtschaft und Gesellschaft ausschlaggebend, um offene Innovation als Hochschule zu leben.

Neben der Anwendungsorientierung wurden einige Merkmale der fachhochschulischen Forschung bereits in [9] und [6] identifiziert und näher analysiert: Geographisch betrachtet, pflegen die FHs neben den hochschultypischen internationalen Netzwerken in Lehre und Forschung auch ganz spezifische regionale Kooperationen mit Wirtschaft, Zivilgesellschaft und Technologietransferintermediären, sodass ihre umsetzungsorientierten Forschungsergebnisse relativ rasch in die Region gelangen. Unter den Kooperationspartnern

${ }^{3}$ Wir bedanken uns an dieser Stelle beim anonymen Reviewerteam für die wertvollen Kommentare!
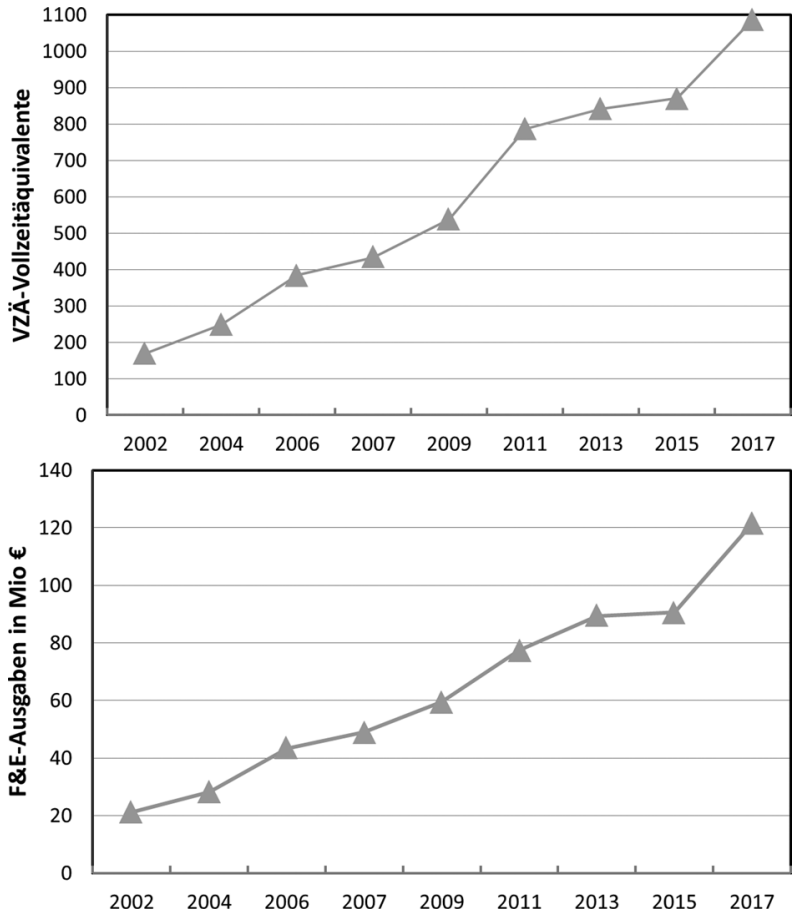

Abb. 1. (a) Vollzeitäquivalente pro Jahr und (b) F\&E Ausgaben in Mio. $€$ (Quelle: Statistik Austria)

der FHs finden sich nicht nur Wissenschaftsplayer und forschende Großunternehmen, sondern auch zahlreiche Kleine und Mittlere Unternehmen (KMUs), die zum Beispiel durch die Innovationsschecks der FFG oder analoge Instrumente der regionalen Förderagenturen (z.B. zum Aufbau von Prototypen) Zugang zur Forschung bekommen. Viele Forschungsthemen, weil sehr anwendungsnah, sind an FHs interdisziplinär sowohl in der Fragestellung als auch in der Bearbeitung und resultieren in laufende, inkrementelle Innovationen. Und: die Verzahnung zwischen Lehre und Forschung ist besonders ausgeprägt, in beiden Richtungen.

\section{Entwicklung}

Die Geschichte vom F\&E Aufbau an den österreichischen FHs ist in den letzten zwanzig Jahren von durchgängigem Wachstum gezeichnet. War das F\&E Volumen im Jahr 2002 insgesamt über alle Institutionen noch ca. 20 Mio. €, sind es im Jahr 2017 bereits über 121 Mio. $€$. Parallel dazu (siehe Abb. 1) stieg die Anzahl der Vollzeitäquivalente auf ca. 1.000 insgesamt mit über 2.700 Beschäftigten in F\&E. ${ }^{4}$ Von 434 Vollzeitäquivalente in F\&E im Jahr 2007 auf 1085 im Jahr 2017, weist der FH Sektor ein Wachstum von $150 \%$ in 10 Jahren. Mit einem jährlichen Wachstum von 9,6 \% pro Jahr ist somit der FH Sektor einer der am stärksten wachsenden F\&E Bereiche in Österreich

\subsection{Finanzierung}

Von den insgesamt 121,3 Mio. € F\&E Volumina an österreichischen FHs im Jahr 2017, kamen ca. 88 Mio. € aus dem öffentlichen Sektor, die restlichen Anteile setzten sich wie in Abb. 2 dargestellt zusammen. Zum Anteil des öffentlichen Sektors trug der Bund 46,3 Mio.

${ }^{4}$ Quelle: Statistik Austria, Erhebung über Forschung und Experimentelle Entwicklung 2017. 


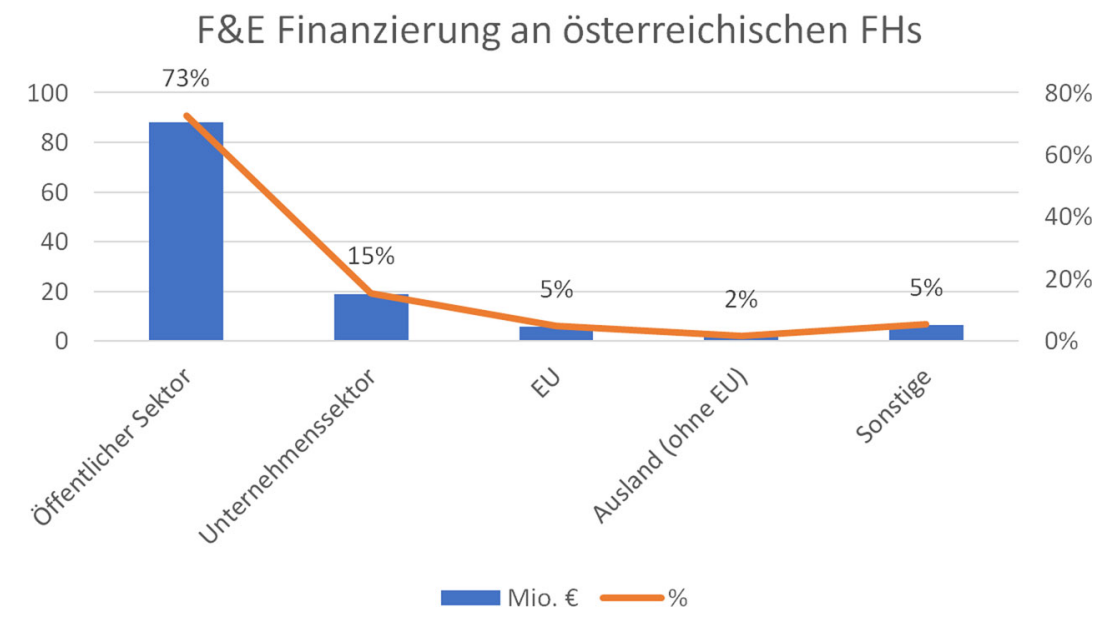

Abb. 2. F\&E Finanzierungsquellen für das Jahr 2017, absolut und relativ (Quelle: Statistik Austria)

F\&E Volumina (Mio. €) an Fachhochschulen in Ö

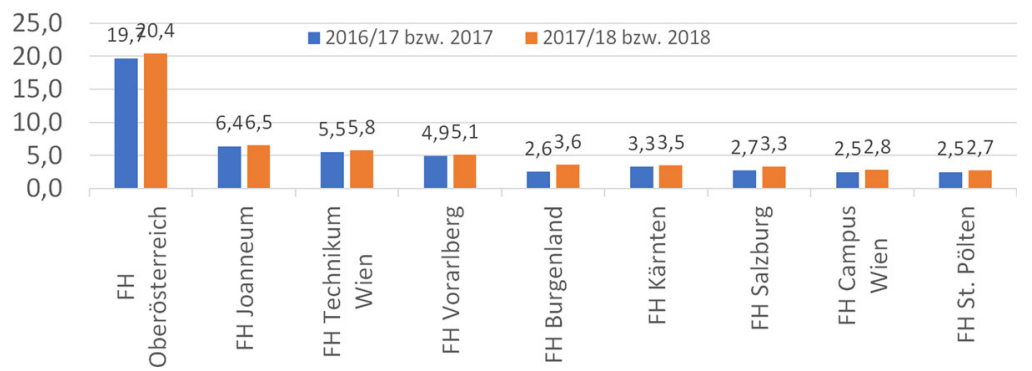

Abb. 3. F\&E Volumina in Mio. € je FH für die Jahre 2016/17 bzw. 2017 und 2017/18 bzw. 2018 (siehe Fußnote 6)

$€(52 \%$ vom Anteil des öffentlichen Sektors und $38 \%$ vom Gesamtfinanzierungsvolumen) und die Länder 25,3 Mio. € (28\% vom Anteil des öffentlichen Sektors und $20 \%$ vom Gesamtbetrag) bei.

Im Erhebungsjahr 2018 gab es insgesamt 1450 Kooperationen mit Firmen im F\&E Bereich, davon $62 \%$ mit KMUs. ${ }^{5}$ Laut [1] machen Unternehmen bezüglich F\&E an FHs ca. $35 \%$ der Kooperationspartner und ca. $14 \%$ der Finanzierung aus.

\subsection{Profilbildung}

Für die letzten zwei verfügbaren Jahre (Studienjahr 2016/17 oder Kalenderjahr 2017 und Studienjahr 2017/18 oder Kalenderjahr 2018, je nach Berechnungszeitraum der jeweiligen Institutionen), zeigt Abb. 3 die aktuelle Entwicklung an den forschungsstärksten $\mathrm{FHs}^{6}$ :

Wie bereits in [4] und [5] festgestellt, ist es mehreren forschungsstarken FHs in Österreich in den letzten zehn bis fünfzehn Jahren gelungen, signifikante und sichtbare F\&E Aktivitäten umzusetzen und kritische Masse in einigen Gebieten aufzubauen. Dies ist umso überraschender angesichts der herausfordernden finanziellen und strukturellen Rahmenbedingungen. Neben der Vorreiterrolle der FH Oberösterreich, wird der zweitgrößte Anteil von der FH Joanneum geleistet, laut [5] machten diese zwei Institutionen im Zeitraum 20142016 zusammen 45 \% des Gesamtfinanzierungsvolumen aus. An

\footnotetext{
${ }^{5}$ Quelle: Daten der FHK, eigene Erhebungen.
}

${ }^{6}$ Quelle: eigene Erhebung der Autoren. Bei den Daten der FH Joanneum handelt es sich um Erlöse, wobei diese Tatsache keinen Einfluss auf die Position in der Reihenfolge hat. dritter Stelle gemessen am F\&E Volumen konnte sich seit einigen Jahren die FH Technikum Wien etablieren. Je nach Betrachtungszeitraum folgen dann Hochschulen aus dieser Gruppe in unterschiedlicher Reihenfolge: FH Vorarlberg, FH Kärnten, FH Salzburg und FH Burgenland. Auf den fünf forschungsstärksten Institutionen entfallen über $65 \%$ des Gesamtfinanzierungsvolumens für F\&E [5].

Einige dieser forschungsstarken FHs konnten in den letzten Jahren auch Kooperationen mit in- und ausländischen Universitäten aufbauen, um gemeinsam Doktorate anzubieten. Unter anderem seien hier im nationalen Kontext die langjährige Zusammenarbeit zwischen der Johannes Kepler Universität Linz und der FH Oberösterreich, sowie das erste kooperative Doktoratskolleg "Resilient Embedded Systems" der Technischen Universität Wien und der FH Technikum Wien als Beispiele genannt. Im internationalen Kontext gibt es unter anderem ein Rahmenabkommen zwischen der FH Vorarlberg und der Universität Agder in Norwegen, das auch ein PhD Programm beinhaltet.

\subsection{Fokussierung}

Dieselbe Quelle gibt Aufschluss darüber, welche Rolle der Bereich Elektrotechnik, Elektronik und Informationstechnik dabei spielt: dieser deckt einen Anteil von $15 \%$ des Gesamtvolumens und liegt unter den Top 3 neben Informatik und neben der Kategorie "andere technische Wissenschaften" (u.a. Werkstofftechnik, Medizintechnik, Nanotechnologie, Verfahrenstechnik). Die höchsten relativen Anteile von Elektrotechnik, Elektronik, Informationstechnik am jeweiligen Forschungsumfang bei den einzelnen Institutionen finden sich bei FH Joanneum, FH Technikum Wien, FH Kärnten und FH St. 


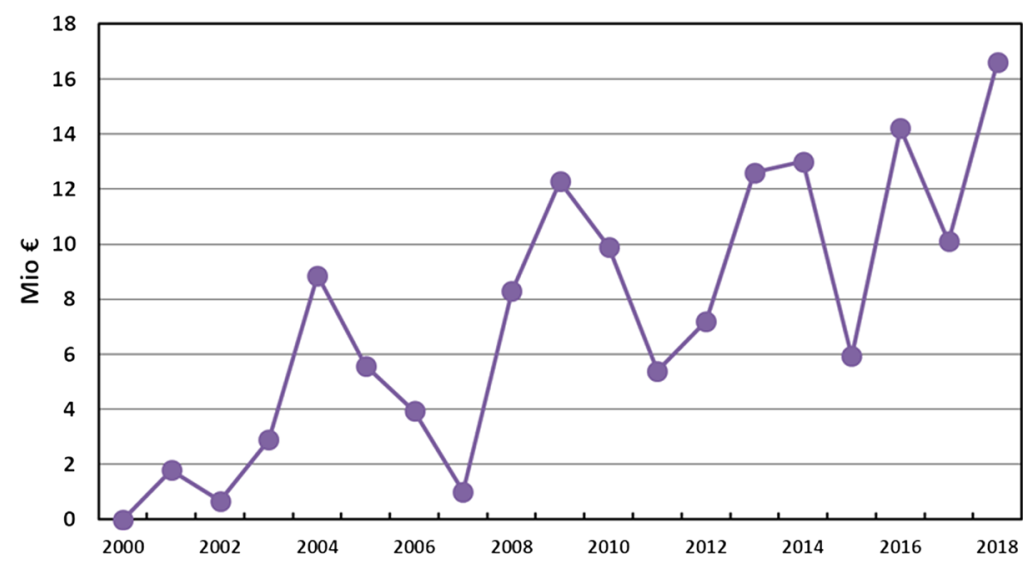

Abb. 4. FFG-Förderzusagen an FHs in Mio. € pro Jahr [3]

Pölten [5]. Unter den zwischen 2010 und 2015 von der Österreichischen Forschungsförderungsgesellschaft (FFG) und von der CDG geförderten Projekten an Fachhochschulen, fielen $19 \%$ der bewilligten Fördermittel auf die Kategorien IKT-Anwendungen, Elektronik und Mikroelektronik und stellen somit den größte Anteil dar [4]. Der Bericht [7] fand außerdem: „Kooperationen zwischen Hochschulen und Unternehmen finden sich überproportional häufig bei Unternehmen der Elektro-, Elektronik-, Fahrzeugbau- und Maschinenbauindustrie, sowie in wissensintensiven Dienstleistungssektoren".

\subsection{Fördergeber}

Im Abschn. 2.1 haben wir bereits die Zusammensetzung der öffentlichen Mittel für F\&E an österreichischen Fachhochschulen dargestellt. Wenn man sich aus den Bundes- und EU-Mitteln die stärksten Fördergeber anschaut, dann stehen die FFG, der Wissenschaftsfonds (FWF), die CDG, und das jeweilige EU-Rahmenprogramm an erster Stelle. Der Bund trug, wie oben angegeben, im Jahr 2017 46,3 Mio. $€$, die EU insgesamt (nicht nur im Rahmenprogramm) 5,8 Mio. $€$.

Bei den Mitteln aus CDG-Förderungen entsteht ca. die Hälfte nicht aus der Finanzierung des Bundes, sondern aus der Beteiligung von Unternehmen und somit aus den oben angegebenen 18,8 Mio. $€$ des Unternehmenssektors. Die Landesfinanzierungsformen fallen dagegen sehr unterschiedlich aus, nicht nur im Hinblick auf ihre Höhe, sondern auch bezüglich der Mechanismen: Von der projektbezogenen Förderung (in unterschiedlichem Ausmaß) bis hin zur strukturellen Unterstützung (z.B. für Kauf, Bau, Leasing, ... verschiedener Infrastruktur), vom Incentive-Mechanismus bei der Akquisition von Drittmitteln bis hin zur Finanzierung bestimmter Stellen und Aktivitäten. Somit müssen diese von der vorliegenden Analyse fürs Erste ausgeschlossen werden.

Wenn wir bei den Fördergebern FFG, FWF, CDG und EURahmenprogramm bleiben, zeigen die Auswertungen aus [5] für die Periode 2014-2016, dass 57 \% der Drittmittel von der FFG, 19 \% von der CDG (wobei fast die Hälfte davon aus dem Unternehmenssektor stammt), $16 \%$ vom EU-Rahmenprogramm und $8 \%$ vom FWF kamen.

Besonders interessant sind daher die näheren Auswertungen der FFG Zahlen, da diese den größten Anteil ausmachen. Laut [3] ergänzt mit früheren Daten, schaut die Entwicklung der vertraglich fixierten Förderzusagen an FHs in den letzten Jahren wie in Abb. 4 dargestellt aus. Für das Jahr 2014 waren dies knapp 13 Mio. €; 2015 fast 6 Mio. €; 2016 über 14 Mio. €; 2017 ca. 10 Mio. € und 2018 16,6 Mio. $€$.

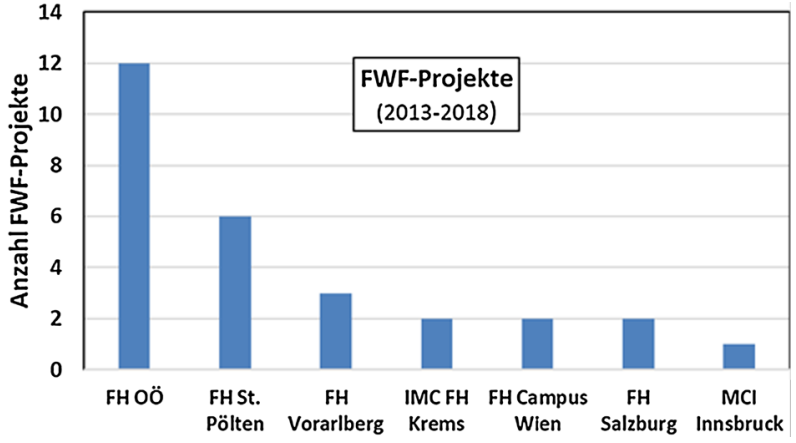

Abb. 5. Anzahl der FWF-Projekte je FH im Zeitraum 2013-2018 (Datenquelle: www.fwf.ac.at).

Woher kommen die starken Schwankungen in diesem Bild? Die Erklärung ist leicht gefunden, wenn man die Beträge pro FFG Förderschiene anschaut: Allein aus dem Programm COIN ergeben sich bis zu 50 \% der FFG Fördermittel pro Jahr für die FHs - aber: COIN, vor allem COIN Aufbau, wurde in den letzten Jahren nicht jährlich und vor allem nicht mit konstanten Budgets ausgeschrieben, was den Hauptgrund für die großen Schwankungen darstellt. Die "nächstwichtigsten" Förderschienen machen zwischen 7 und $8 \%$ der FFG Förderzusagen für FHs (e!MISSION, Mobilität der Zukunft, TAKE OFF), bzW. zwischen 5 und 6 \% (Forschungskompetenzen für die Wirtschaft, benefit, Bridge) aus. Die Rolle von COIN für die Finanzierung der FH Forschung braucht hier nicht weiter unterstrichen zu werden.

Ein Blick auf Abb. 5 bezüglich der FWF Förderungen für $\mathrm{FHs}^{7}$ zeigt, dass dieser Fördergeber trotz aller Anstrengungen nach wie vor für wenige FH-Projekte in Frage kommt. Sowohl der FWF als auch die FHs haben in den letzten Jahren Maßnahmen umgesetzt, um diesen Trend umzukehren, da es durchaus Forschungsbereiche an FHs gibt, die im Förderportfolio vom FWF reüssieren könnten.

Sehr positiv schaut hingegen die Situation bezüglich Josef Ressel Zentren aus, die FH-spezifische Förderschiene der CDG nach dem Vorbild und den Erfahrungen aus den Christian Doppler Laboren für Universitäten. Die Anzahl der angepeilten JRZ bis 2020 konnte bereits vorzeitig erreicht werden, wie Abb. 6 zeigt. Die Suche nach exzellenter FH-Forschung im Spagat zwischen Unternehmens-

\footnotetext{
${ }^{7}$ Quelle: Project Finder auf www.fwf.ac.at.
} 


\section{Aktive JRZ}

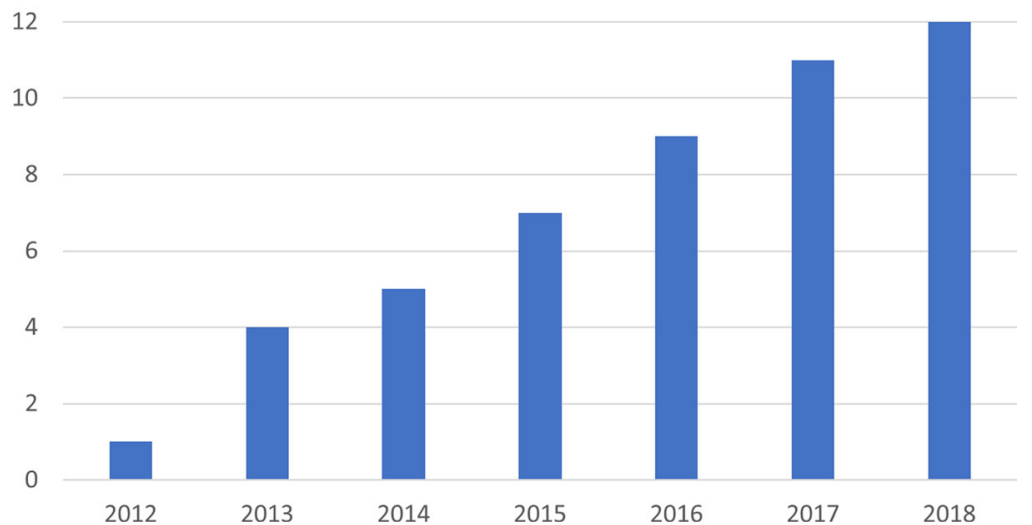

Abb. 6. Anzahl der aktiven Josef Ressel Zentren pro Jahr (Datenquelle: www.cdg.ac.at)

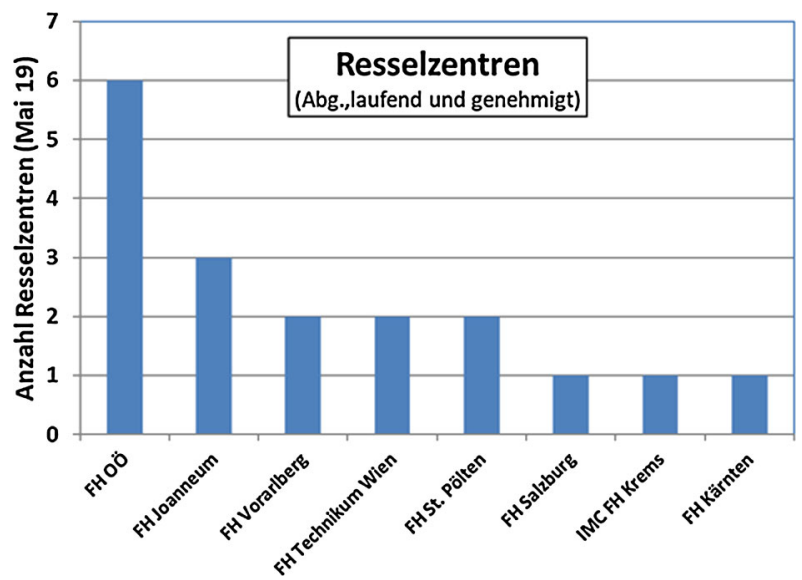

Abb. 7. Gesamtanzahl Josef Ressel Zentren je FH (Datenquelle: www.cdg.ac.at Stand Mai 2019)

kooperationen und wissenschaftlicher Tiefe ist mittlerweile acht FHs gelungen (siehe Abb. 7)

Laufend waren im Jahr 2018 drei JRZ an der FH Oberösterreich, zwei jeweils an der FH Joanneum, FH Vorarlberg und FH Technikum Wien, ein JRZ je an der FH St. Pölten, FH Krems und FH Kärnten entstanden. Insgesamt hat der Bund im Jahr 2018 2,7 Mio. $€$ in diese Förderschiene investiert. Von den bisher eingerichteten JRZ waren 7 aus dem thematischen Cluster "Mathematik, Informatik, Elektronik" [2].

Was das laufende EU-Rahmenprogramm Horizon 2020 betrifft, gibt es derzeit 18 Beteiligungen von österreichischen FHs, allen voran die FH Burgenland mit über 1,2 Mio. $€$ Gesamtförderungen. $^{8}$ Darüber hinaus wichtige internationale Finanzierungsquellen für $\mathrm{FHs}$ sind auch die Mittel aus dem Europäischen Fonds für regionale Entwicklung (EFRE), zum Beispiel aus dem Programm IWB (Investitionen in Wachstum und Beschäftigung, 29 Beteiligungen per Juni 2019) und aus den verschiedenen Interreg-Programmen (zum Beispiel allein im Programm Interreg Österreich - Bayern 2014-2020 finden sich 9 Projekte mit österreichischer FH-Beteiligung). ${ }^{9}$

\footnotetext{
${ }^{8}$ Quelle: www.ffg.at/monitoring Stand März 2019.

${ }^{9}$ Quellen: https://www.interreg-bayaut.net/projekte/liste-der-vorhaben/ fü die Liste der Vorhaben - Projekte INTERREG Österreich - Bayern 2014 - 2020
}

\section{Rahmenbedingungen und Diskussion}

Die hier zusammengefassten Zahlen und Entwicklungen zeigen eindrucksvoll, dass der Aufbau der F\&E Leistung an den österreichischen Fachhochschulen eine Erfolgsgeschichte ist. Inwieweit sind die bestehenden Rahmenbedingungen förderlich oder hinderlich für den weiteren Auf- und Ausbau, für die Profilbildung der jeweiligen Institutionen, für die Weiterentwicklung eines wichtigen Teils des gesamten Hochschulsektors, für dessen Leistungen für die österreichische Gesellschaft und den wissenschaftlichen und wirtschaftlichen Standort? Einige Antworten auf diese Frage werden in diesem Abschnitt untersucht.

Es ist keine neue Erkenntnis, dass die Hauptschwierigkeit im bisherigen Auf- und Ausbau von Forschungsleistung an den Fachhochschulen an der mangelnden nachhaltigen bzw. strategischen zentralen Finanzierung seitens des Bundes liegt. Damit Institutionen Forschungskapazitäten, Know-how, Infrastruktur, Sichtbarkeit auf bestimmten Gebieten aufbauen können, die dann förder- und auftragswürdig werden, und neben sowie zwischen Förderprojekten und Aufträgen weiterhin finanziert, leistungs- und ausbaufähig bleiben, braucht es strategisches Geld. Die Erhalter können diese Finanzierung nur bedingt bzw. begrenzt zur Verfügung stellen oder aus anderen (regionalen oder in der Wirtschaft angesiedelten) Quellen akquirieren. Das ist der gravierendste Engpass im Aufbau und in der Erhaltung von F\&E an österreichischen FHs. Der Forschungsauftrag, der im FHStG formuliert wurde, ist per se nicht finanziert. Öfter haben verschiedene Player im Hochschul- und Innovationssystem in den letzten Jahren auf dieses Problem aufmerksam gemacht und verschiedene Ansätze, wie eine nachhaltige Finanzierung des Bundes ausschauen könnte, um weder "nach dem Gießkannenprinzip", noch eine reine projektbezogene Förderung zu beinhalten, sind ausgearbeitet worden. Zu einer Umsetzung ist es noch nicht gekommen.

Umgekehrt: „COIN Aufbau“, die Förderschiene aus der bestehenden FH-Forschungsförderung, die am ehesten einen strategischen Strukturaufbau bisher ermöglicht hat und sehr positiv evaluiert wurde [4], ist in den letzten Jahren den Änderungen der Förderlogik zum Teil zum Opfer gefallen. Ausschreibungen sind nicht mehr jährlich möglich, die Budgets der einzelnen Ausschreibungen sinken - obwohl die Anzahl an potenziellen Forschungsgruppen im FH-Sektor,

und https://www.efre.gv.at/projekte/ für die Projektlandkarte EFRE / IWB2020, Stand Juli 2019. 
die von dieser Aufbauförderung profitieren könnte, laufend steigt. Folgt man der Diagnose in [4], „[...] sind die Möglichkeiten für Fachhochschulen, im Falle von Mittelreduktionen bei diesen beiden Programmen [COIN-Aufbau und JR-Zentren] kurzfristig auf andere Förderschienen oder Fördergeber auszuweichen beschränkt". Wie sollen diese Gruppen wettbewerbsfähig werden können, wenn sie vom Anfang an mit erfahreneren, größeren, breit aufgestellten Forschungsgruppen und -anträgen in Konkurrenz sind? Planbarkeit und signifikante Volumina sind hier gefragt, damit diese Förderschiene in Zukunft weiterhin ihre wichtige Rolle in der FH Forschungsfinanzierung erfüllen kann.

Rein projektbezogene Finanzierungen sind oft relativ kurz, decken gerade ein bis zwei Jahre ab, beinhalten keine vor- und nachbereitende Projektphase, ermöglichen keinen nachhaltigen Aufbau von Personal, Know-how, Infrastruktur, bereiten der Institution nachgelagerte Kosten zum Beispiel für die Instandhaltung bzw. Aufrechterhaltung von Forschungsinfrastruktur, hinterlassen Lücken in der Personalfinanzierung und verlangen eine immer kurzfristigere Anpassung von Themen und Inhalten an die Bedürfnisse der Auftraggeber oder des Fördermarkts.

Vor dem Hintergrund fehlender Aufbaufinanzierung, erscheint es umso erstaunlicher, dass es mehreren Institutionen gelungen ist, in der Exzellenz-Förderschiene für FHs, die JRZ der CDG, erfolgreich zu werden. Damit ein Antrag genehmigt (und später der Projektverlauf positiv begutachtet) wird, müssen hier zwei nicht leicht zu vereinbarenden Bedingungen erfüllt werden: das Interesse der Wirtschaft an verwertbaren Forschungsergebnissen muss mehrere Unternehmen überzeugen, sich zu etwa $50 \%$ an der Projektfinanzierung zu beteiligen (die zweite Hälfte kommt aus Bundesförderungen über die $(D G) ;$ gleichzeitig müssen der Antrag und der Werdegang der antragstellenden Person wissenschaftlich exzellent sein und im Lauf des Projektes zu qualitativ hochwertigen wissenschaftlichen Publikationen führen. Dieser Spagat ist per se nicht leicht zu schaffen, da in der Praxis diese zwei Kriterien unterschiedliche Ansprüche an Projektplanung und -durchführung stellen, umso schwieriger, wenn die beantragende Person und die dahinterstehende Forschungsgruppe ihren Aufbau aufgrund mangelnder nachhaltiger Finanzierung nicht strategisch anlegen können.

Neben den in vielen Fällen befristeten Beschäftigungsverhältnissen von Forschenden und dem steigenden Druck im nationalen und internationalen Wettbewerb um Drittmittel [7], erschwert eine andere Rahmenbedingung den österreichischen FHs den Aufbau und die Höherqualifikation ihrer Personalressourcen: Anders als traditionelle Universitäten, haben die FHs kein Promotionsrecht. Während andere Länder hier bereits Modelle entwickelt haben, wie entweder FHs zu (akkreditierten) Doktoratsprogrammen auf bestimmten Gebieten (in bestehenden forschungsstarken Bereichen) kommen können oder kooperative Doktorate zwischen Universitäten und FHs aufgesetzt werden können, ist das in Österreich noch nicht der Fall. Während schon länger an der Entwicklung eines Modells für kooperative Doktorate gearbeitet wird (siehe auch [1] und die darin für 2019 in Aussicht gestellte entsprechende Ausschreibung), haben einige FHs bereits erste Erfahrungen gesammelt, unter anderem sowohl die FH Oberösterreich in Zusammenarbeit mit der Johannes Kepler Universität Linz als auch die FH Technikum Wien mit einem gemeinsamen Doctoral Program on Resilient Embdedded Systems mit der Technischen Universität Wien. Weiterhin soll bei der Ausgestaltung der Chancen für junge Forschende und DissertantInnen auch die Europäische Charter für Forscherlnnen im Auge behalten werden, die den Umgang, die Professionalisierung, die Anstellung, die Rekrutierung und Weiterbildung dieser für das Innovationssystem so wichtigen Personengruppe gut regelt.
Ebenfalls wünschenswert wäre die Möglichkeit für FHs, sich wie die traditionellen Universitäten an den Ausschreibungen des Bundes für Stiftungsprofessuren zu beteiligen. Einige Institutionen haben in der Vergangenheit bereits ihr Interesse mitgeteilt, trotz Gesprächen ist diese Fördermöglichkeit nach wie vor den Universitäten vorbehalten. Dass das Modell der Stiftungsprofessuren für FHs den Aufbau gut mitunterstützen kann, zeigen unter anderem die Stiftungsprofessuren (und Kompetenzteams) der Stadt Wien, die seit Jahren ein erfolgreiches Fördermodell darstellen.

Bezüglich der aktuellen Förderlandschaft lässt sich im Hinblick auf die österreichischen FHs folgendes zusammenfassen:

- In Anbetracht der Rolle, welche die FFG Förderungen für die FHs spielen, steigen die FFG Budgets nicht in ausreichendem Maß. Diversifizierung wäre gefragt, unter anderem auch mehr Erfolg bei den FWF Ausschreibungen, dies ist ebenfalls noch nicht in ausreichendem Ausmaß gelungen. Das Förderprogramm „Translational Research", das bis vor einigen Jahre mehr Raum für FH Forschung beim FWF anbot, ist nicht mehr vorhanden und wird in nächster Zeit planmäßig nicht ersetzt bzw. neu aufgebaut

- Die Möglichkeit, JRZ bei der CDG zu beantragen und aufzubauen, wurde dagegen sehr erfolgreich aufgegriffen. Hier bleibt es abzuwarten, wie der längerfristige Trend sich entwickelt und ob die hohe Anzahl an JRZ gehalten werden kann. Die Empfehlung der OECD [8] ist hier eindeutig: „Continue with the successful CDG model of funding industry-university cooperation which combines [...] basic research with industrial application, providing all partners with powerful incentives to co-operate, and assuring the quality of research performed in the CD Laboratories and JR Centres".

- Für COIN Aufbau braucht es mehr Planungssicherheit im Hinblick auf die Ausschreibungszeiträume und höhere Budgets pro Ausschreibung, nur so kann dieses Förderprogramm seine wichtige Rolle für den FH Sektor weiterhin erfüllen.

- Nicht zu vergessen sind jene Forschungsgebiete, die traditionell weniger vom bestehenden Fördersystem adressiert werden und zum Teil schwieriger Anschluss an die Auftragsforschung finden, u.a. zum Beispiel rund um die Gesundheitsberufe. Wenn Österreich das Innovationspotenzial der FHs auch auf diesen für die Gesellschaft und die Wirtschaft wichtigen Gebieten nutzen will, sind hier eigene Instrumente und Modelle zu entwickeln.

- Im europäischen Rahmenprogramm (RP, derzeit Horizon 2020) gelingt es derzeit den FHs nicht, an den Erfolgschancen aus dem RP 7 anzuschließen. Trotz tiefergehenden Analysen seitens der FFG und der Institutionen im Rahmen der FHK, konnten hier noch keine zielführenden Maßnahmen identifiziert und umgesetzt werden.

Der Beobachtung und Empfehlung aus [4] möchten wir uns an dieser Stelle anschließen: „Es sollte vermieden werden, Fachhochschulen direkt oder indirekt (noch weiter) in die Auftragsforschung zu drängen. Auftragsforschung bedeutet in der Realität sehr häufig Kleindienstleistungen, die keinen strukturell wirksamen Wissens- und Know-how-Aufbau an den Fachhochschulen zulassen".

\section{Ausblick}

Welche Rahmenbedingungen bräuchten die österreichischen FHs, um weiterhin am nationalen und internationalen Forschungs- und Innovationssystem erfolgreich zu sein und ihren Wachstumskurs qualitativ und quantitativ fortzusetzen? Zwei sind aus unserer Sicht unabdingbar: 
- Eine nachhaltige Forschungsfinanzierung seitens des Bundes hat höchste Priorität, Kriterien und Modelle sollten in Zusammenarbeit mit dem zuständigen Ministerium ausgearbeitet werden.

- Neben der Finanzierungsfrage sind die Chancen einer Höherqualifikation im Rahmen von Doktoratsprogrammen für $\mathrm{FH}$ Forschende strukturell extrem wichtig. Akkreditierte Programme oder kooperative Doktorate - eine tragfähige Lösung in signifikantem Ausmaß, inklusive Finanzierung, ist für den gesamten Sektor erforderlich. Wir finden, dass die Empfehlung der OECD [8] „Expand modern doctoral schools with structured PhD training and improve funding for PhDs" auch in Kooperation mit Fachhochschulen umgesetzt werden sollte.

Neben den strukturellen Erfordernissen kommen weitere Herausforderungen auf die österreichischen FHs zu:

- Gelingt es dem Bund, genügende Finanzmittel aufzustellen, steht dem FH Sektor in den nächsten Jahren weiterhin ein Wachstum bevor, in erster Linie bei den Studierendenzahlen. Dieses Wachstum bedeutet, dass noch mehr qualifiziertes Lehr- und Forschungspersonal erforderlich sein wird (siehe auch [8]), auch in Bereichen wo in Österreich gut qualifiziertes Personal chronisch Mangelware ist und die FHs immer wieder aufgefordert werden, mehr AbsolventInnen zu generieren. Wie wird es gelingen, die erforderliche Qualität in Lehre und Forschung zu gewährleisten?

- Dabei dürfen FHs nicht ihre Identität aus den Augen verlieren, bei der die enge Verschränkung von Lehre und Forschung ein Unterscheidungsmerkmal im Hochschulsektor darstellt.

- Der Wettbewerb um Fördermittel für die Forschung und um Firmenkooperationen wird immer schwieriger. Wie sollen und können sich die FHs insgesamt und die einzelnen Institutionen in ihrer Strategie und Profilbildung weiterentwickeln und positionieren, um hier sichtbar und konkurrenzfähig zu bleiben?

- Weiterhin aufrecht bleibt eben die institutionsspezifische Frage nach der Positionierung und Profilbildung. Eine Ausdifferenzierung ist auch unter den FHs weiterhin sinnvoll.

- Die Gesamtfrage der Akademisierung und des Forschungsaufbaus rund um andere in der Förderlandschaft unterrepräsentierten Disziplinen ist ebenfalls noch offen.

- Trotz einigen Nachbesserungen wird das nächste Europäische Rahmenprogramm für F\&E, Horizon Europe, sich entlang der gleichen Dimensionen wie Horizon 2020 entwickeln. Angesichts der enttäuschenden Erfolgszahlen der FHs in der letzten Förderperiode, bleibt die strategische Frage offen, wie man wieder wettbewerbsfähig(er) im europäischen Förderkontext wird. Hier wäre ev. eine nationale Initiative in Zusammenarbeit mit dem Wissenschaftsressort und der FFG sinnvoll.

Unter anderem interessant wäre für die Zukunft eine Vertiefung der Analyse der Forschungsleistung. Strukturen, Kooperationen, Modelle wären hier von großem Interesse für den gesamten FH Sektor und möglicherweise für mehrere Fördergeber. Solche Studien wären neben den Evaluierungen von einzelnen Förderprogrammen wichtig für eine strategische Weiterentwicklung. Die Daten zeigen, dass sowohl bei der Vereinheitlichung der Erhebungsmethoden und der Quellen, als auch bei Tiefe und Detailgrad, sowie bei der Sammlung von qualitativen Informationen noch Bedarf gegeben ist. Darauf ließen sich im und mit dem Sektor kriti- sche Interpretationen und Diskussionen begründen. Ebenfalls interessant wären Analysen der Länderspezifika, vor allem im Hinblick auf Finanzierung und Governance-Mechanismen rund um die Forschung.

\section{Danksagung}

Die Autoren möchten sich sowohl bei der FHK als auch bei einzelnen FHs für die Bereitstellung von Daten bedanken.

Hinweis des Verlags Der Verlag bleibt in Hinblick auf geografische Zuordnungen und Gebietsbezeichnungen in veröffentlichten Karten und Institutsadressen neutral.

Open Access Dieser Artikel wird unter der Creative Commons Namensnennung 4.0 International Lizenz veröffentlicht, welche die Nutzung, Vervielfältigung, Bearbeitung, Verbreitung und Wiedergabe in jeglichem Medium und Format erlaubt, sofern Sie den/die ursprünglichen Autor(en) und die Quelle ordnungsgemäßnennen, einen Link zur Creative Commons Lizenz beifügen und angeben, ob Änderungen vorgenommen wurden. Die in diesem Artikel enthaltenen Bilder und sonstiges Drittmaterial unterliegen ebenfalls der genannten Creative Commons Lizenz, sofern sich aus der Abbildungslegende nichts anderes ergibt. Sofern das betreffende Material nicht unter der genannten Creative Commons Lizenz steht und die betreffende Handlung nicht nach gesetzlichen Vorschriften erlaubt ist, ist für die oben aufgeführten Weiterverwendungen des Materials die Einwilligung des jeweiligen Rechteinhabers einzuholen. Weitere Details zur Lizenz entnehmen Sie bitte der Lizenzinformation auf http://creativecommons.org/licenses/by/4.0/deed.de.

\section{Literatur}

1. BMBWF (2019): Fachhochschulentwicklungs- und Finanzierungsplan 2018/192022/23. Wien: Bundesministerium für Bildung, Wissenschaft und Forschung (BMBWF). https://bmbwf.gv.at/fileadmin/user_upload/wissenschaft/publikationen/FH_Plan_ 201819.pdf.

2. Ecker, B., Brunner, P., Christmann-Budian, S., Fischl, I., Gassler, H., Gogola, G., Hartmann, E., Heckl, E., Kaufmann, P., Krabel, S., Mayer, K., Mozhova, A., Pechar, H., Radauer, A., Reiner, C., Ruhland, S., Sardadvar, S., Schneider, H. W., Schuch, K., Sturn, D., Tiefenthaler, B., Warta, K., Welp-Park, E. (2019): Österreichischer Forschungsund Technologiebericht 2019. Wien: im Auftrag der Bundesministerien für Bildung, Wissenschaft und Forschung (BMBWF), Verkehr, Innovation und Technologie (BMVIT), Digitalisierung und Wirtschaftsstandort (BMDW). https://www.bmvit.gv.at/service/ publikationen/innovation/forschungsberichte/downloads/ftb 2019.pdf.

3. FFG (2019): Österreichische Fachhochschulen in nationalen FFG-Programmen und in Horizon 2020. Wien: Österreichische Forschungsförderungsgesellschaft mbH, EU-PM.

4. Gever, A., Warta, K. (2015): Evaluierung der Forschungsförderung für Fachhochschulen in Österreich. Wien: Technopolis Group im Auftrag des Bundesministeriums für Wissenschaft, Forschung und Wirtschaft (BMWFW). https://repository.fteval.at/18/1/ Evaluierung \%20der\%20Forschungsf\%C3\%B6rderung\%20f\%C3\%BCr\%20Fachhoch schulen $\% 20$ in $\% 20 \%$ C3\%96sterreich.pdf.

5. Heller-Schuh, B., Zahradnik, G., Leitner, K.-H. (2018): Forschung an Fachhochschulen: Analyse forschungsrelevanter Fachhochschuldaten. Wien: AIT-ISP-Report 14, Austrian Institute of Technology (AIT) im Auftrag des Bundesministeriums für Bildung. Wissenschaft und Forschung (BMBWF). https://repository.fteval.at/393/1/Endbericht $\% 20 F H 20$ Forschung final rev.pdf.

6. Kastner, J. (2014): Forschung an Fachhochschulen. In H. Holzinger, K. Koleznik (Hrsg.), 20 Jahre Fachhochschulen in Österreich - Rolle und Wirkung, Wien: Facultas.

7. Leitner, K.-H., Dachs, B., Degelsegger, A., Ecker, B., Gassler, H., Heller-Schuh, B., Hochgerner, J., Janger, J., Lampert, D., Peneder, M., Ploder, M., Polt, W., Schemgell, T., Schuch, K., Streicher, G., Unger, M., Unterlass, F., Zahradnik, G. (2015): Stärkefelder im Innovationssystem: Wissenschaftliche Profilbildung und wirtschaftliche Synergien. Wien: Austrian Institute of Technology (AIT) im Auftrag des Bundesministeriums für Wissenschaft, Forschung und Wirtschaft (BMWFW). AIT-IS-Report. https://bmbwf.gv.at/fileadmin/user_upload/wissenschaft/publikationen/forschung/AT_ Forschungsraum_Endbericht.pdf.

8. OECD (2018): OECD reviews of innovation policy: Austria 2018. Paris: OECD Publishing. https://doi.org/10.1787/9789264309470-en

9. ÖHZ spezial (2012): Forschung \& Entwicklung an Österreichs Fachhochschulen. Kastner J. (Hrsg.). Wien; F\&E-Ausschuss der Österreichischen Fachhochschulkonferenz. 


\section{Autoren}

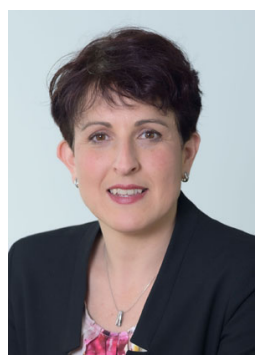

\section{Giuliana Sabbatini}

ist seit 2010 Leiterin der Forschungsorganisation an der FH Technikum Wien und Mitglied des F\&E Ausschusses der FHK, sowie Vertreterin der FHK in der RDI-Arbeitsgruppe von UAS4Europe. Sie studierte Computer Science an der Universität "La Sapienza” in Rom und promovierte 2002 an der Technischen Universität Wien auf dem Gebiet der Wissensbasierten Systemen. Von 1997 bis 1999 und von 2002 bis 2009 war sie als Unternehmensberaterin in Wien tätig, vor allem in den Bereichen Strategieberatung, Team- und Organisationsentwicklung, DWH- und CRM-Beratung, und in der Leitung von großen IT- und Organisationsprojekten. Sie ist im Auftrag der Europäischen Kommission als Trainerin und Assessor für den HRS4RProzess tätig - Human Resources Strategy for Research / HR Excellence in Research zur Umsetzung der Prinzipien der European Charter \& Code for Researchers.

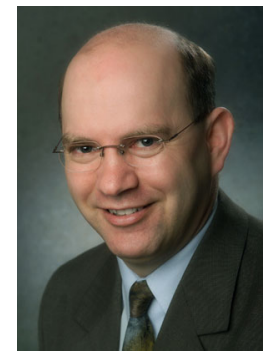

\section{Johann Kastner}

ist seit 2002 Professor für Messtechnik an der Fachhochschule OÖ und seit 2004 Leiter der F\&E der FH OÖ. Weitere F\&E-relevante Funktionen sind Vorsitzender des F\&E-Ausschusses der FHK (Österreichische Fachhochschulkonferenz) seit 2007, Mitglied des Rates Forschung und Technologie OÖ von 2009 2019, Mitglied des Forschungs- und Wissenschaftsrates Kärnten seit 2019, Mitglied im Josef Ressel Senat der Christian Doppler Gesellschaft seit 2017 und diverse Aufsichtsratsfunktionen (TCKT -Transferzentrum für Kunststofftechnik, RECENDT - Research Center for non-destructive Testing GmbH, K1-Zentrum für Lebensmittel - FFOQSI Austrian Competence Centre for Feed and Food Quality, Safety \& Innovation). Er studierte Technische Physik an der Universität Linz, promovierte an der Universität Wien und habilitierte sich für das Fachgebiete „'Zerstörungsfreie Prüfung und Computertomografie" an der TU Wien. Nach dem Studiumabschluss arbeitete er als Entwicklungsingenieur bei iR3 Video International Wien und als Forschungsleiter und Geschäftsführer bei der Profactor Produktionsforschungs $\mathrm{GmbH}$, bevor er 2002 an die FH OÖ kam. 